PROCEEDINGS OF THE

AMERICAN MATHEMATICAL SOCIETY

Volume 127, Number 6, Pages 1589-1595

S 0002-9939(99)04689-4

Article electronically published on February 18, 1999

\title{
CYCLIC TORSION OF ELLIPTIC CURVES
}

\author{
TETSUO NAKAMURA
}

(Communicated by William W. Adams)

Dedicated to Professor Tsuneo Kanno on his seventieth birthday

\begin{abstract}
Let $E$ be an elliptic curve over a number field $k$ such that $\operatorname{End}_{k} E$ $=\mathbf{Z}$ and let $w(k)$ denote the number of roots of unity in $k$. Ross proposed a question: Is $E$ isogenous over $k$ to an elliptic curve $E^{\prime} / k$ such that $E^{\prime}(k)_{\text {tors }}$ is cyclic of order dividing $w(k)$ ? A counter-example of this question is given. We show that $E$ is isogenous to $E^{\prime} / k$ such that $E^{\prime}(k)_{\text {tors }} \subset \mathbf{Z} / w(k)^{2} \mathbf{Z}$. In case $E$ has complex multiplication and $\operatorname{End}_{k} E=\mathbf{Z}$, we obtain certain criteria whether or not $E$ is isogenous to $E^{\prime} / k$ such that $E^{\prime}(k)_{\text {tors }} \subset \mathbf{Z} / 2 \mathbf{Z}$.
\end{abstract}

\section{INTRODUCTION}

Let $k$ be a field finitely generated over its prime field, and let $w(k)$ denote the number of roots of unity in $k$. For an elliptic curve $E$ over $k$, Ross [2] proposed the following question:

Question 1. If $\operatorname{End}_{k} E=\mathbf{Z}$, then is $E$ isogenous over $k$ to an elliptic curve $E^{\prime} / k$ such that $E^{\prime}(k)_{\text {tors }}$, the $k$-rational torsion points of $E^{\prime}$, is cyclic of order dividing $w(k)$ ?

In this paper we assume that $k$ is a finite extension of the rationals $\mathbf{Q}$. An example in Section 4 shows that Question 1 does not hold in general. Put $D=$ $\operatorname{Min}\left|E^{\prime}(k)_{\text {tors }}\right|$, where the minimum is taken over those $E^{\prime} / k$ which are isogenous to $E$ over $k$. We will show in Section 2 that if $\operatorname{End}_{k} E=\mathbf{Z}$, then $D \mid w(k)^{2}$ and $E$ is isogenous over $k$ to an elliptic curve $E^{\prime} / k$ such that $E^{\prime}(k)_{\text {tors }}$ is cyclic of order $D$ (Theorem 1). In Section 3 we treat an elliptic curve $E$ over $k$ with $\operatorname{End}_{k} E=\mathbf{Z}$, but which has complex multiplication by an imaginary quadratic field $K$. We will prove that $E$ is $k$-isogenous to $E^{\prime} / k$ such that $E^{\prime}(k)_{\text {tors }} \subset \mathbf{Z} / 4 \mathbf{Z}$. We will also give certain criteria for $E^{\prime}$ to be chosen so that $E^{\prime}(k)_{\text {tors }} \subset \mathbf{Z} / 2 \mathbf{Z}$ (Theorem 2, Proposition 1 ). In Section 4 we will give some examples concerning other questions in Ross [2].

Notations: For a number field $k$ and a prime number $p$, denote by $w(k)\left(w_{p}(k)\right.$, resp.) the number of roots ( $p$ th-power roots, resp.) of unity in $k$. The Galois

Received by the editors December 11, 1996 and, in revised form, September 8, 1997.

1991 Mathematics Subject Classification. Primary 11G05.

Key words and phrases. Elliptic curve, torsion point, isogeny, complex multiplication.

The author was supported by Grant-Aid for Scientific Research No. 09640003, Ministry of Education, Science and Culture, Japan.

(c)1999 American Mathematical Society 
$\operatorname{group} \operatorname{Gal}(\bar{k} / k)$ is simply denoted by $G_{k}$ or $G(\bar{k} / k)$. If $A$ is an abelian group, we denote by $A[n]$ the subgroup of $A$ annihilated by $n$. For an elliptic curve $E$ over $k$, the $p$-Sylow subgroup of $E(k)_{\text {tors }}$ is denoted by $E(k)_{(p)}$. We write $\mathcal{C}(E)$ for the $k$-isogeny class of $E$. End $E$ is the endomorphism ring of $E$ and $\operatorname{End}_{k}$ is the subring of End $E$ consisting of endomorphisms defined over $k$.

\section{Minimal CyClic torsion}

Let $E$ be an elliptic curve over $k$. For a prime number $p$, we denote by $T_{p}(E)$ the $p$-adic Tate module of $E$ and by $\rho$ the corresponding representation of $G_{k}$ on $T_{p}(E)$.

Lemma 1. There exists an elliptic curve $E^{\prime}$ in $\mathcal{C}(E)$ such that $E$ has an isogeny over $k$ onto $E^{\prime}$ of p-power degree such that $E^{\prime}(k)_{(p)}$ is cyclic.

Proof. If $\operatorname{End}_{k} E=\mathbf{Z}$, then $V_{p}(E)=T_{p}(E) \otimes \mathbf{Q}_{p}$ is irreducible as a $G_{k}$-space (see [3, IV $\S 2]$ ). If $E$ has complex multiplication by an imaginary quadratic field $K$ over $k$, then $V_{p}(E)$ is reducible if and only if $p$ splits in $K$.

(1) The case $V_{p}(E)$ is reducible. We may assume that End $E$ is isomorphic to the maximal order of $K$. Then the decomposition $K \otimes \mathbf{Q}_{p}=\mathbf{Q}_{p} \oplus \mathbf{Q}_{p}$ gives that of $T_{p}(E)=T_{1} \oplus T_{2}$ as a $G_{k}$-module. Therefore we have representations $\chi_{i}: G_{k} \rightarrow$ Aut $T_{i}=\mathbf{Z}_{p}^{\times}(i=1,2)$ and $\rho=\chi_{1} \oplus \chi_{2}$. The theory of complex multiplication shows that $\operatorname{Im} \rho$ is open in $\mathbf{Z}_{p}^{\times} \times \mathbf{Z}_{p}^{\times}$. Hence we can choose an integer $n$ such that for all $\sigma \in G_{k}$

$$
\chi_{1}(\sigma) \equiv \chi_{2}(\sigma) \quad \bmod p^{n}
$$

and for some $\sigma \in G_{k}$,

$$
\chi_{1}(\sigma) \not \equiv \chi_{2}(\sigma) \bmod p^{n+1} .
$$

Let $T^{\prime}=\mathbf{Z}_{p} e_{1}+\mathbf{Z}_{p} \frac{e_{1}+e_{2}}{p^{n}}$, where $T_{i}=\mathbf{Z}_{p} e_{i}$. Then $T^{\prime}$ is a $G_{k}$-module and we obtain an elliptic curve $E^{\prime} / k$ and an isogeny $f: E \rightarrow E^{\prime}$ defined over $k$ with $\operatorname{Ker} f=\left\langle e_{1}+e_{2} \bmod p^{n}\right\rangle$. Let $\rho^{\prime}$ be the $p$-adic representation associated with $E^{\prime}$. Then we get

$$
\rho^{\prime}(\sigma) \equiv\left(\begin{array}{cc}
\chi_{1}(\sigma) & 0 \\
u & \chi_{2}(\sigma)
\end{array}\right) \quad \bmod p^{n}, \quad u \neq \equiv \quad \bmod p .
$$

This implies that $E^{\prime}(k)_{(p)}$ is cyclic.

(2) The case $V_{p}(E)$ is irreducible. In this case it is clear that $\mathbf{Z}_{p} x$ is not $G_{k}$-stable for a basis $\{x, y\}$ of $T_{p}(E)$. Thus there is $\sigma \in G_{k}$ such that

$$
x^{\sigma}=a x+p^{r} u y, \quad u \neq \equiv 0 \quad \bmod p\left(a \in \mathbf{Z}_{p}\right) .
$$

We choose $r(\geq 0)$ minimal under this condition. Then since the subgroup $\langle x \bmod$ $\left.p^{r}\right\rangle$ of $E\left[p^{r}\right]$ is $G_{k^{-}}$-stable, $T^{\prime}=\mathbf{Z}_{p} p^{-r} x+\mathbf{Z}_{p} y$ is $G_{k^{-}}$-stable. Then as in (1), $T^{\prime}$ defines $E^{\prime} \in \mathcal{C}(E)$ such that $E^{\prime}(k)_{(p)}$ is cyclic. This proves our assertion.

Lemma 2. Assume that $V_{p}(E)$ is irreducible. If $E$ contains a $k$-rational point of order $p^{2 n+1}$ with $p^{n}=w_{p}(k)$, then there is $E^{\prime} \in \mathcal{C}(E)$ such that $E^{\prime}(k)_{(p)} \subset \mathbf{Z} / p^{n} \mathbf{Z}$.

Proof. By assumption, we may assume that

$$
\rho\left(G_{k}\right) \subset\left(\begin{array}{cc}
1+p^{2 n+1} \mathbf{Z}_{p} & \mathbf{Z}_{p} \\
p^{2 n+1} \mathbf{Z}_{p} & \mathbf{Z}_{p}^{\times}
\end{array}\right) .
$$


Let $\tau \in G_{k}$ be such that $\tau$ induces a generator in $G\left(k\left(\zeta_{p^{n+1}}\right) / k\right)$, where $\zeta_{p^{n+1}}$ is a primitive $p^{n+1}$ th root of unity. Since $\operatorname{det} \rho$ is the $p$-adic cyclotomic character of $G_{k}$, we can write

$$
\rho(\tau)=\left(\begin{array}{cc}
1+p^{2 n+1} a & b \\
p^{2 n+1} c & d
\end{array}\right)
$$

where $a, b, c, d \in \mathbf{Z}_{p}$ and $d \equiv 1 \bmod p^{n}$ and $d \not \equiv 1 \bmod p^{n+1}$. The characteristic polynomial $f(t)$ of $\rho(\tau)$ satisfies $f(t) \equiv(t-1)(t-d) \bmod p^{2 n+1}$. Putting $t=1+p^{n} s$, we have

$$
p^{-2 n} f(t) \equiv s\left(s-p^{-n}(d-1)\right) \quad \bmod p .
$$

By Hensel's lemma, $f(t)$ has solutions $\lambda_{1}, \lambda_{2} \in \mathbf{Z}_{p}$ such that $\lambda_{1} \equiv 1, \lambda_{2} \equiv d \bmod$ $p^{n+1}$. We can choose $P=\left(\begin{array}{cc}1 & b^{\prime} \\ p^{n+1} c^{\prime} & p^{n}\end{array}\right) \quad\left(b^{\prime}, c^{\prime} \in \mathbf{Z}_{p}\right)$ such that $P^{-1} \rho(\tau) P=$ $\left(\begin{array}{cc}\lambda_{1} & 0 \\ 0 & \lambda_{2}\end{array}\right)$. Choose a basis $\left\{x_{1}, x_{2}\right\}$ of $T_{p}(E)$ such that $x_{1} \bmod p^{2 n+1}$ is a $k$ rational point of order $p^{2 n+1}$. Put $y_{1}=x_{1}+p^{n+1} c^{\prime} x_{2}$ and $y_{2}=b^{\prime} x_{1}+p^{n} x_{2}$. Then the lattice $T^{\prime}=\mathbf{Z}_{p} y_{1}+\mathbf{Z}_{p} y_{2}=\mathbf{Z}_{p} x_{1}+p^{n} \mathbf{Z}_{p} x_{2}$ is $G_{k^{-}}$stable and $T^{\prime}$ determines an elliptic curve $E^{\prime} \in \mathcal{C}(E)$ such that $\rho^{\prime}(\tau)=\left(\begin{array}{cc}\lambda_{1} & 0 \\ 0 & \lambda_{2}\end{array}\right)$, where $\rho^{\prime}$ is the $p$-adic representation associated with $E^{\prime}$. Since $T^{\prime}$ is irreducible, there is $\sigma \in G_{k}$ such that $\rho^{\prime}(\sigma)=\left(\begin{array}{cc}\alpha & * \\ p^{r} u & \beta\end{array}\right)$, with $u \not \equiv 0 \bmod p$ and $r \geq 1$. Choose $r$ minimal under the above condition. Put $T^{\prime \prime}=\mathbf{Z}_{p} y_{1}^{\prime}+\mathbf{Z}_{p} y_{2}$ with $y_{1}^{\prime}=p^{-r} y_{1}$. Then $T^{\prime \prime}$ also defines $E^{\prime \prime} \in \mathcal{C}(E)$ and the correponding representation $\rho^{\prime \prime}$ satisfies

$$
\rho^{\prime \prime}(\tau)=\left(\begin{array}{cc}
\lambda_{1} & 0 \\
0 & \lambda_{2}
\end{array}\right), \rho^{\prime \prime}(\sigma) \equiv\left(\begin{array}{cc}
\alpha & * \\
u & \beta
\end{array}\right) \quad \bmod p
$$

We see that $E^{\prime \prime}(k)_{(p)}$ is cyclic. Let $P=a y_{1}^{\prime}+b y_{2} \bmod p^{n+1}$. Then $P=P^{\tau}$ and $P=P^{\sigma}$ implies $a \equiv 0, b \equiv 0 \bmod p$. This shows that $E^{\prime \prime}(k)$ does not contain any point of order $p^{n+1}$. This completes the proof.

Lemma 3. Put $p^{n}=w_{p}(k)$ and $p^{m}=\operatorname{Min}_{E^{\prime} \in \mathcal{C}(E)}\left|E^{\prime}(k)_{(p)}\right|$. If $V_{p}(E)$ is irreducible, then $m \leq 2 n$ and there exists $E^{\prime} \in \mathcal{C}(E)$ such that $E^{\prime}(k)_{(p)}$ is cyclic of order $p^{m}$.

Proof. The first assertion follows from Lemma 2. Hence we may assume that

$$
E(k)_{(p)} \cong \mathbf{Z} / p^{r} \mathbf{Z} \oplus \mathbf{Z} / p^{r^{\prime}} \mathbf{Z}, \quad 1 \leq r \leq r^{\prime}, r+r^{\prime}=m \leq 2 n .
$$

Choose a basis $\{x, y\}$ of $T_{p}(E)$ such that $x \bmod p^{r}$ and $y \bmod p^{r^{\prime}}$ are $k$-rational points of $E$. As in the proof of Lemma 1, there exists an integer $s(\geq r)$ such that the subgroup $X=\left\langle x \bmod p^{s}\right\rangle$ is $G_{k^{-}}$-stable and $\left\langle x \bmod p^{s+1}\right\rangle$ is not $G_{k^{-}}$stable; if $r=r^{\prime}$, changing $x$ and $y$ if necessary, we may assume that $\left\langle y \bmod p^{s}\right\rangle$ is $G_{k}$-stable. Putting $E^{\prime}=E / X$, we obtain $E^{\prime} \in \mathcal{C}(E)$ such that $E^{\prime}(k)_{(p)}$ is cyclic. It suffices to show that each point $P$ in $E^{\prime}(k)_{(p)}$ is of order dividing $p^{m}$. We may suppose that $T_{p}\left(E^{\prime}\right)=\mathbf{Z}_{p} x^{\prime}+\mathbf{Z}_{p} y$ with $x^{\prime}=p^{-s} x$. A point $P$ of $E^{\prime}\left[p^{m+1}\right]$ can be written in the form $\alpha x^{\prime}+\beta y \bmod p^{m+1}$. 
(i) First assume $s>r$. Since $x \bmod p^{r+1}$ is not $k$-rational in $E$, there is $\sigma \in G_{k}$ such that $x^{\sigma} \equiv x+p^{r} v x \bmod p^{r+1}$ with $v \in \mathbf{Z}_{p}^{\times}$. If $r\left\langle n\right.$, then $\operatorname{det} \rho(\sigma) \equiv 1 \bmod p^{n}$ shows that $r=r^{\prime}$, and with respect to the basis $\left\{x^{\prime}, y\right\}, \rho^{\prime}(\sigma)$ takes the form

$$
\left(\begin{array}{cc}
1+p^{r} v & p^{r+s} b \\
c & 1+p^{r} v^{\prime}
\end{array}\right) \quad\left(v^{\prime} \equiv-v \bmod p\right)
$$

If $P^{\sigma}=P$, then

$$
p^{r} v \alpha \equiv 0, c \alpha+p^{r} v^{\prime} \beta \equiv 0 \bmod p^{m+1} .
$$

Therefore $\alpha \equiv \beta \equiv 0 \bmod p$. If $r=n$, then $r=r^{\prime}$ and there is $\sigma \in G_{k}$ such that $y^{\sigma^{\prime}} \equiv y+p^{r} u y \bmod p^{r+1}$ with $u \in \mathbf{Z}_{p}^{\times}$. Clearly $P^{\sigma}=P$ and $P^{\sigma^{\prime}}=P$ imply $\alpha \equiv \beta \equiv 0 \bmod p$.

(ii) Next assume $s=r$. In this case there is $\sigma \in G_{k}$ such that $x^{\sigma}=x+p^{r} a x+p^{r} u y$ with $u \in \mathbf{Z}_{p}^{\times}$. If $P^{\sigma}=P$, then $u \alpha+p^{r^{\prime}} d \beta \equiv 0 \bmod p^{m+1}$, which shows that $\alpha x^{\prime}+\beta y=\gamma x+\beta y\left(=z\right.$, say) with $\gamma \in \mathbf{Z}_{p}$. Now $z^{\sigma} \equiv z \bmod p^{m+1} T_{p}\left(E^{\prime}\right)$ for $\sigma \in G_{k}$ implies $z^{\sigma} \equiv z \bmod p^{r^{\prime}+1} T_{p}(E)$, because $p^{m+1} T_{p}\left(E^{\prime}\right) \subset p^{r^{\prime}+1} T_{p}(E)$. As $E(k)$ contains no point of order $p^{r^{\prime}+1}$, we have $\alpha \equiv \beta \equiv 0 \bmod p$. Therefore every point of $E^{\prime}(k)_{(p)}$ is of order dividing $p^{m}$. This proves our assertion.

Theorem 1. Put $D=\operatorname{Min}_{E^{\prime} \in \mathcal{C}(E)}\left|E^{\prime}(k)_{\text {tors }}\right|$. If $\operatorname{End}_{k} E=\mathbf{Z}$, then $D$ is a divisor of $w(k)^{2}$ and there exists an elliptic curve $E^{\prime}$ in $\mathcal{C}(E)$ such that $E^{\prime}(k)_{\text {tors }}$ is cyclic of order D.

Proof. Let $p$ be a prime divisor of $\left|E(k)_{\text {tors }}\right|$. By the above three lemmata, $E$ is $k$-isogenous to $E^{\prime}$ of $p$-power degree such that $E^{\prime}(k)_{(p)}$ is cyclic of the minimal $p$-power order. Repeating this process we obtain Theorem 1.

\section{ElLiptic CURVES With COMPLEX MULTiPlicAtion}

Let $E$ be an elliptic curve over $k$ with complex multiplication by an imaginary quadratic field $K$ and suppose that $k \not \supset K$.

Lemma 4 (Aoki [1, Theorem 9.1]). If $w_{p}(k)=w_{p}(K k)$, then $E(k)_{(p)}=0$ for $p>$ 2 and $E(k)_{(2)} \subset \mathbf{Z} / 2 \mathbf{Z}$ for $p=2$.

Proof. For the sake of completeness we sketch the proof in [1]. By Tchebotarev's density theorem, there is a prime ideal $\mathfrak{l}$ of $k$ satisfying the following conditions:

- $(\mathfrak{l}, p)=1$ and $E$ has good reduction modulo $\mathfrak{l}$.

- The Frobenius automorphism associated with $\mathfrak{l}$ in $G\left(K k\left(\zeta_{p^{n+1}}\right) / k\right)$ acts trivially on $\zeta_{p^{n+1}}$ and non-trivially on $K$.

Then we have $|\tilde{E}(F)|=1+N \mathfrak{l}$ and $N \mathfrak{l} \equiv 1 \bmod p^{n+1}$, where $\tilde{E}$ is the reduction of $E$ modulo $\mathfrak{l}$ over the residue field $F$ and $N \mathfrak{l}$ is the norm of $\mathfrak{l}$. Since $N \mathfrak{l}+1 \equiv 2 \bmod$ $p^{n+1}$, we see that $|\tilde{E}(F)|$ is prime to $p$ for $p>2$ and $4 \backslash\langle\tilde{E}(F)|$ for $p=2$. As the reduction map $E(k) \rightarrow \tilde{E}(F)$ is injective, we get our assertion.

Theorem 2. Let $E$ be an elliptic curve over $k$ with complex multiplication by an imaginary quadratic field $K$ and suppose that $k \not \supset K$, i.e. $\operatorname{End}_{k} E=\mathbf{Z}$. Then $E$ is $k$-isogenous to an elliptic curve $E^{\prime} / k$ such that $E^{\prime}(k)_{\text {tors }} \subset \mathbf{Z} / 4 \mathbf{Z}$. If $K k \neq k(\sqrt{-1})$, then $E^{\prime}$ can be chosen such that $E^{\prime}(k)_{\text {tors }} \subset \mathbf{Z} / 2 \mathbf{Z}$. 
Proof. Since $[K k: k]=2, p \leq w_{p}(k)\left\langle w_{p}(K k)\right.$ never happens for $p>2$. Hence by Theorem 1 and Lemma $4, E$ is isogenous to an elliptic curve $E^{\prime}$ over $k$ such that $E^{\prime}(k)_{\text {tors }}$ is cyclic of 2-power order. If $K k=k(\sqrt{-1})$, then $w_{2}(k)<w_{2}(K k)$, which implies $w_{2}(k)=2$. Therefore we get the first assertion by Lemma 3. Now assume that $K k \neq k(\sqrt{-1})$. If $w_{2}(k)=w_{2}(K k)$, our assertion follows from Lemma 4 . Hence we may further assume that $w_{2}(k)<w_{2}(K k)$. This implies that $\sqrt{-1} \in k$. Let $\tau$ be an element of $G_{k}$ which acts non-trivially on $K$. Then $\alpha \rightarrow \alpha^{\tau}(\alpha \in$ End $E$ induces a non-trivial automorphism of End $E$. Let $\rho: G_{k} \rightarrow \operatorname{Aut}\left(T_{2}(E)\right)$ be the 2-adic representation associated with $E$. As $\alpha^{\tau}=\rho(\tau) \alpha \rho(\tau)^{-1}, \rho(\tau)$ induces the non-trivial automorphism of $K \otimes \mathbf{Q}_{2}=$ End $E \otimes \mathbf{Q}_{2}$. Then $\rho(\tau)$ is conjugate to a matrix of the form $\left(\begin{array}{cc}a & b \\ m b & -a\end{array}\right)$ where $K=\mathbf{Q}(\sqrt{-m})$ and $a, b \in \mathbf{Z}_{2}$. By Lemma 1, we may assume that $E(k)_{(2)}$ is cyclic. Now $\sqrt{-1} \in k \operatorname{implies} \operatorname{det} \rho(\tau) \equiv 1 \bmod 4$. If $E(k)$ contains a point of order 4 , then $\rho(\tau)$ is conjugate to a matrix of the form $\left(\begin{array}{ll}1 & * \\ 0 & 1\end{array}\right) \bmod 4$. Since $\operatorname{tr} \rho(\tau)=0$, this is a contradiction. Therefore $E(k)_{(2)} \subset$ $\mathbf{Z} / 2 \mathbf{Z}$.

Proposition 1. Let $E / k, K$ be as in Theorem 2. Assume $K k=k(\sqrt{-1})$. Let $H(f)$ denote the ring class field of $K$ of conductor $f$ and choose $n$ maximal such that $H\left(2^{n}\right) \subset K k$. Assume further that 4||$E(k)_{\text {tors }} \mid, E(K k) \supset \mathbf{Z} / 4 \mathbf{Z}$, and for any $E^{\prime} \in \mathcal{C}(E)$, End $E^{\prime}$ has conductor not divisible by $2^{n}$. Then for any $E^{\prime} \in \mathcal{C}(E)$, we have $E^{\prime}(k) \supset \mathbf{Z} / 4 \mathbf{Z}$ or $E^{\prime}(k) \supset E^{\prime}[2]$.

Proof. We divide the proof into three steps.

Step 1. $E^{\prime}(K k) \supset \mathbf{Z} / 2 \mathbf{Z} \otimes \mathbf{Z} / 2 \mathbf{Z}$ for any $E^{\prime} \in \mathcal{C}(E)$.

Proof of Step 1. Let $j^{\prime}$ be the $j$-invariant of $E^{\prime}$. If $j^{\prime}=0$, then $K=\mathbf{Q}(\sqrt{-3})$ and $E^{\prime}$ is isomorphic to the curve $y^{2}=x^{3}+a, a \neq 0$. As $E^{\prime}(k)_{(2)} \neq 0$, our assertion is clear. If $j^{\prime}=1728$, then $K=\mathbf{Q}(\sqrt{-1})$ and $E^{\prime}$ is isomorphic to the curve $y^{2}=x^{3}+a x$. If $E^{\prime}(K k) \not \supset \mathbf{Z} / 2 \mathbf{Z} \otimes \mathbf{Z} / 2 \mathbf{Z}$, then $x^{2} \pm a=0$ has no solutions in $K k$, hence $E^{\prime}(K k) \cong \mathbf{Z} / 2 \mathbf{Z}$. Let $f: E^{\prime} \rightarrow E^{\prime \prime}$ be a $k$-isogeny of degree 2. Since $\operatorname{Ker} f=\langle(0,0)\rangle, E^{\prime \prime}$ is $K k$-isomorphic to the curve $y^{2}=x\left(x^{2}-4 a\right)$. Then we also have $E^{\prime \prime}(K k)_{(2)}=\mathbf{Z} / 2 \mathbf{Z}$. This shows that $E^{\prime}(K k)_{(2)}=\mathbf{Z} / 2 \mathbf{Z}$ for all $E^{\prime} \in \mathcal{C}(E)$. This contradicts the condition 4||$E(k)_{\text {tors }} \mid$. Now assume that $j^{\prime} \neq 0,1728$. Let the conductor of End $E^{\prime}$ be $2^{r} d,(2, d)=1$. Then we have

$$
H\left(2^{r} d\right)=K\left(j^{\prime}\right), \quad K k \supset H\left(2^{r} d\right) H\left(2^{n}\right) \supset H\left(2^{r+1} d\right) .
$$

Since we have $H\left(2^{r+1} d\right)=K\left(j^{\prime}, E^{\prime}[2]\right)$ by [4, Theorem 5.5], we get that $E^{\prime}(K k) \supset$ $\mathbf{Z} / 2 \mathbf{Z} \otimes \mathbf{Z} / 2 \mathbf{Z}$

Step 2. $E^{\prime}(K k) \supset \mathbf{Z} / 4 \mathbf{Z}$ for any $E^{\prime} \in \mathcal{C}(E)$.

Proof of Step 2. It suffices to show that for an isogeny $f: E \rightarrow E^{\prime}$ defined over $k$ of degree $2, E(K k) \supset \mathbf{Z} / 4 \mathbf{Z}$ implies $E^{\prime}(K k) \supset \mathbf{Z} / 4 \mathbf{Z}$. Let $P$ be a $K k$-rational point of $E$ of order 4 . If $\operatorname{Ker} f \neq\langle 2 P\rangle$, then $f(P)$ is a $K k$-rational point of $E^{\prime}$ of order 4. If $\operatorname{Ker} f=\langle 2 P\rangle$, then $f\left(P^{\prime}\right)$ is a $K k$-rational point of $E^{\prime}$ of order 4 , where $P^{\prime} \in E[4]$ is such that $2 P^{\prime} \neq 2 P$.

Step 3. For $E_{1}, E_{2} \in \mathcal{C}(E)$, let $f: E_{1} \rightarrow E_{2}$ be an isogeny over $k$ of degree 2. If $\left|E_{1}(k)_{(2)}\right| \geq 4$, then $\left|E_{2}(k)_{(2)}\right| \geq 4$. 
Proof of Step 3. Let $\operatorname{Ker} f=\langle e\rangle$, where $\left\{e, e^{\prime}\right\}$ is a basis of $E_{1}[2]$. Assume that $E_{1}(k)_{(2)}$ is cyclic. Then $E_{1}(k)$ contains a point $P$ of order 4 . If $2 P \neq e$, our assertion is clear. If $2 P=e$, then $E_{2}(k) \supset\left\langle f(P), f\left(e^{\prime}\right)\right\rangle=E_{2}$ [2]. Next assume that $E_{1}(k) \supset E_{1}[2]$. Let $P, P^{\prime}$ be such that $2 P=e, 2 P^{\prime}=e^{\prime}$. Since $E(K k) \supset$ $\mathbf{Z} / 4 \mathbf{Z} \otimes \mathbf{Z} / 2 \mathbf{Z}$, we may assume that $P$ or $P^{\prime} \in E_{1}(K k)$. If $P^{\sigma} \neq P$ for some $\sigma \in G_{K k}$, then $\operatorname{det} \rho(\sigma) \equiv 1 \bmod 4$ shows that $P^{\sigma}=P+e^{\prime}$. Hence $f(P)$ is not $K k$-rational, which contradicts Step 1. Thus we get $P \in E_{1}(K k)$. Similarly if $P^{\prime \sigma} \neq P^{\prime}$ for some $\sigma \in G_{K k}$, we have $P^{\prime \sigma}=P^{\prime}+e$ and this implies that $E_{1}^{\prime}(K k) \not \supset E_{1}^{\prime}[2]$ with $E_{1}^{\prime}=E_{1} /\left\langle e^{\prime}\right\rangle$. Consequently we have $E_{1}(K k) \supset E_{1}[4]$. Let $\langle\tau\rangle=G(K k / k)$. Since $\operatorname{det} \rho(\tau) \equiv-1 \bmod 4$, we obtain

$$
P^{\tau}=a P+m e^{\prime}, \quad P^{\prime \tau}=b P^{\prime}+n e
$$

with $a b \equiv-1 \bmod 4$. If $b=1$, then $f\left(P^{\prime}\right)$ is a $k$-rational point of order 4 . If $b=-1$ and $m=0$, then $\left\langle f(P), f\left(e^{\prime}\right)\right\rangle=E_{2}[2] \subset E_{2}(k)$. If $b=-1$ and $m=1$, then we have $\left(P+P^{\prime}\right)^{\tau}=P+P^{\prime}+n e$, which shows that $f\left(P+P^{\prime}\right)$ is a $k$-rational point of order 4 . This completes the proof.

\section{EXAMPLES}

The following is a counter-example of Question 1 in the Introduction.

Example 1. Let $k=\mathbf{Q}(\sqrt{-2})$ and $E: y^{2}=x\left(x^{2}-16\right)$. Notations being as in Proposition 1, we see that $w(k)=2, K=\mathbf{Q}(\sqrt{-1})$ and $K k=H\left(2^{2}\right)$. If $E^{\prime} \in \mathcal{C}(E)$, then $j\left(E^{\prime}\right) \in k$ has a real conjugate, hence $j\left(E^{\prime}\right) \in \mathbf{Q}$ (cf. [4, p.124]). Therefore End $E^{\prime}$ has conductor 1 or 2 . This shows that 4||$E(k)_{\text {tors }} \mid$ for all $E^{\prime} \in \mathcal{C}(E)$ by Proposition 1.

Question 2 ([2]). Let $w_{p}(k)=p^{n}(n \geq 1)$. If $E / k$ contains a $k$-rational point of order $p^{d}$ with $d \leq n$, then does every elliptic curve $E^{\prime} \in \mathcal{C}(E)$ contain a $k$-rational point of order $p^{d}$ ?

If $n \geq 1$ and $d=1$, Question 2 is valid ([2, Proposition 2]). The following example shows that when $p=2, n=d=2$, Question 2 no longer holds.

Example 2. Let $k=\mathbf{Q}(\sqrt{2-2 i})(\ni i=\sqrt{-1})$ and

$$
E: y^{2}=x^{3}-2 x^{2}-x, \quad E^{\prime}: y^{2}=x^{3}+4 x^{2}+8 x .
$$

There is an isogeny $E \rightarrow E^{\prime}$ of degree 2 defined over $\mathbf{Q}$ and $E$ has a $k$-rational point $P=(i, \sqrt{2-2 i})$ of order 4 . Put $c=2-2 i$ and $c^{\prime}=2+2 i$. The points of $E^{\prime}$ of order 2 are

$$
e=(0,0), e_{1}=(-c, 0), e_{2}=\left(-c^{\prime}, 0\right) .
$$

Let $P=(X, Y) \in E^{\prime}$. If $2 P=e$, then $X= \pm 2 \sqrt{2} \notin k$. If $2 P=e_{1}$, then $X=c \pm 2 \sqrt{c^{\prime}} \notin k$. If $2 P=e_{2}$, then $X=c^{\prime} \pm 2 \sqrt{c}$ and $Y= \pm 2 \sqrt{-\sqrt{-1}} X \notin k$. Therefore $E^{\prime}$ has no $k$-rational point of order 4 .

The following example shows that Proposition 5 (hence Question 4) in [2, Section $4]$ is not valid in general.

Example 3. Let $k=\mathbf{Q}(\sqrt[4]{-3}) \supset K=\mathbf{Q}(\sqrt{-3})$ and

$$
E: y^{2}=x^{3}-1, \quad E^{\prime}: y^{2}=x^{3}-6 x^{2}-3 x .
$$

Then $p=2$ is inert in $K$ and $w_{2}(k)=2$. There is an isogeny $E \rightarrow E^{\prime}$ of degree 2 defined over $\mathbf{Q}$ and $E^{\prime}$ has a $k$-rational point $P=(-\sqrt{-3}, \sqrt[4]{-3}(3-\sqrt{-3}))$ of order 
4. We see that $2 \omega P=0$ with $\omega^{2}+\omega+1=0$. But we have $E(k)_{(2)}=E[2]$. Let $E_{1} \in \mathcal{C}(E)$ and put $j_{1}=j\left(E_{1}\right)$. Since $K\left(j_{1}\right) / \mathbf{Q}$ is Galois, we have $K=K\left(j_{1}\right)$ and this implies $j_{1} \in \mathbf{Q}$, hence $j_{1}=j(E)$ or $j\left(E^{\prime}\right)$. It follows that $E_{1}$ is $k$-isomorphic to $E$ or to $E^{\prime}$. Therefore if $E_{1}(k)_{\text {tors }}$ is cyclic for $E_{1} \in \mathcal{C}(E)$, then $E_{1}(k)_{\text {tors }} \supset \mathbf{Z} / 4 \mathbf{Z}$.

\section{REFERENCES}

1. N. Aoki, Torsion points on abelian varieties with complex multiplication, In Algebraic Cycles and Related Topics, Kitasakado 1994, F. Hazama, ed., World Scientific, Singapole, New Jersey, London, HongKong, 1995, 1-22. CMP 97:02

2. R. Ross, Minimal torsion in isogeny classes of elliptic curves, Trans. AMS 344(1994), 203215. MR 95b: 11058

3. J.-P. Serre, Abelian l-adic representations and elliptic curves, Benjamin, New York, 1968.

4. G. Shimura, Introduction to the arithmetic theory of automorphic functions, Iwanami Shoten and Princeton Univ. Press, 1971. MR 95e:11048

Mathematical Institute, Tohoku University, Sendai 980-8578, Japan

E-mail address: nakamura@math.tohoku.ac.jp 